\title{
Controlling Infiltration Pressure of a Nanoporous Silica Gel via Surface Treatment
}

\author{
Aijie Han and Yu Qiao* \\ Department of Structural Engineering, University of California at San Diego, \\ La Jolla, CA 92093-0085, U.S.A.
}

(Received April 17, 2007; CL-070421; E-mail: yqiao@ucsd.edu)

\begin{abstract}
Energy absorption behaviors of a surface-treated nanoporous silica gel are investigated through a pressure-induced infiltration experiment. The results show that the infiltration pressure is dominated by the surface coverage, and thus by controlling the surface treatment time the system performance can be adjusted quite conveniently.
\end{abstract}

While it has been a well-known fact that a certain amount of energy would be dissipated as a liquid flows in a channel, ${ }^{1}$ not until recently with the development of nanoporous technology did this phenomenon receive wide attention for energy absorption applications. For instance, it was noticed that as water moved from compressive parts of a hydrophilic nanoporous silica to tensile parts, considerable energy dissipation took place. $^{2}$ A more attractive way to dissipate energy, however, is based on the capillary effect, for which the nanoporous phase must be lyophobic. ${ }^{3}$ As a nonwetting liquid is forced to enter a nanoporous material, external work is transformed to the excess solid-liquid interfacial tension. Since in a number of systems this process is irreversible, it can be employed for developing advanced protection or damping structures, e.g., liquid armors and liquid bumpers. A key factor dominating the system performance is the specific surface area, which, for most of nanoporous materials, is in the range of $100-2000 \mathrm{~m}^{2} / \mathrm{g}$, six to eight orders of magnitude larger than in bulk materials, resulting in a superior energy absorption efficiency.

Another important design variable of the nanoporous energy absorption system (NEAS) is the degree of lyophobicity, or, if the liquid phase is water based, the degree of hydrophobicity, which is measured by the excess solid-liquid interfacial tension, $\Delta \gamma=\gamma_{\mathrm{sl}}-\left(\gamma_{\mathrm{s}}+\gamma_{1}\right)$, where $\gamma_{\mathrm{sl}}$ is the effective solid-liquid interfacial tension at nanopore surface, and $\gamma_{\mathrm{s}}$ and $\gamma_{1}$ are the effective surface tensions of the solid and the liquid, respectively. On the one hand, a higher degree of hydrophobicity would lead to a higher energy absorption efficiency. On the other hand, $\Delta \gamma$ must be in an appropriate range so that the working pressure is harmless; otherwise the system would be too "rigid," causing blast-lung type problems. ${ }^{4}$ That is, the degree of hydrophobicity must be controlled in a relatively broad range to meet various functional requirements.

In a previous study, it was confirmed that using chemical admixtures can either increase or decrease the infiltration pressure. ${ }^{5}$ However, the addition of admixtures would cause heterogeneous liquid structures, ${ }^{6}$ making it difficult to develop reliable systems, especially when the system must work in a broad temperature range. Therefore, it is desirable to directly control the pore surface structure using surface treatment methods. Compared with ordinary surface treatment, the modification of properties of inner nanopore walls is relatively difficult owing to the poor surface accessibility. The molecular size of the functional agents must be much smaller than the nanopores, and the diffusion process might take a long time. On the other hand, one advantage of NEAS is that leaching is not a concern. Since many protection systems, such as car bumpers and body armors, work only under the first loading, even if the functional groups are deactivated as a high pressure is applied, it would not affect the energy absorption performance.

In order to validate the above considerations, a Davisil236845 nanoporous silica gel was investigated experimentally. The average pore size was $16.2 \mathrm{~nm}$; the specific pore volume was $1.07 \mathrm{~cm}^{3} / \mathrm{g}$; and the specific surface area was $305 \mathrm{~m}^{2} / \mathrm{g}$. The as-received material was in powder form, with the particle size around $250 \mu \mathrm{m}$. The surface treatment was performed in a $100-\mathrm{mL}$ round bottom flask, by the technique discussed by Lim and Stein. ${ }^{7}$ Prior to the treatment, the silica gel was vacuum dried at $100^{\circ} \mathrm{C}$ for $24 \mathrm{~h}$. Immediately after the flask was taken out of the oven, a glass topper was used to seal it. Then, $40 \mathrm{~mL}$ of Sigma-244511 dry toluene was injected into the flask. While the solution was stirred gently, $1.0 \mathrm{~mL}$ of Fluka-92360 chlorotrimethylsilane was injected. The light yellow mixture was stirred for $10 \mathrm{~min}$ at ambient temperature and then refluxed in a hot mantle at $90{ }^{\circ} \mathrm{C}$. The treatment time was in the range of $1-48 \mathrm{~h}$. A drying tube was attached to the reflux apparatus to minimize the influence of moisture. The silica gel was vacuum filtered, washed by dry toluene, and then vacuum dried at $100{ }^{\circ} \mathrm{C}$ for $24 \mathrm{~h}$ to remove the residual chlorotrimethylsilane. Finally, the sample was washed by distilled water so as to hydrolyze remaining chloride groups and dried again in a vacuum oven.

A stainless steel cylinder was sealed by a steel piston with reinforced gasket, which contained $0.5 \mathrm{~g}$ of surface-treated silica gel and $7 \mathrm{~g}$ of distilled water. The piston was compressed by a type 5583 Instron machine at a constant rate of $0.5 \mathrm{~mm} / \mathrm{min}$ into the cylinder, applying a quasi-hydrostatic pressure on the liquid phase. When the pressure exceeded $50 \mathrm{MPa}$, the piston was moved out at the same speed. The loading-unloading cycles were repeated for a few times. The measured sorption isotherm

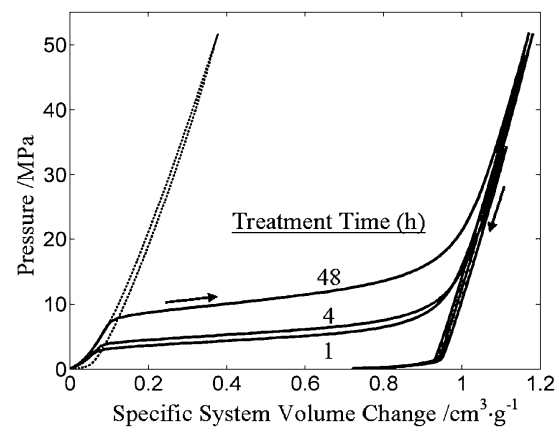

Figure 1. Typical sorption isotherm curves at the first loading. The dashed line indicates the reference curve. 


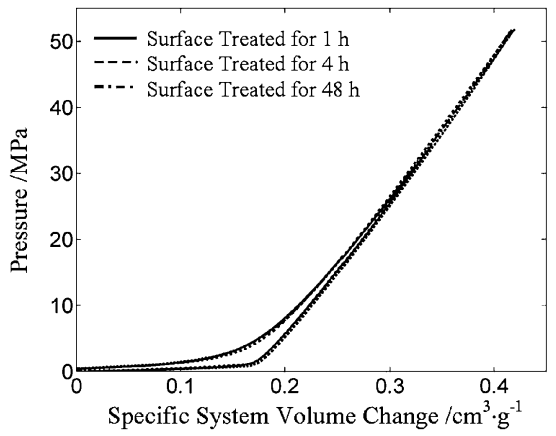

Figure 2. Sorption isotherm curves at the second loading.

curves of the first cycle were shown in Figure 1. The curves of the second cycle were shown in Figure 2. The sorption isotherm curves in the following cycles were nearly identical to the second ones. The specific system volume change was defined as the intrusion volume of the piston divided by the mass of silica gel.

Figure 1 indicates clearly that the surface treatment is quite successful. Without any treatment, the nanopore wall is hydrophilic. Thus, when the silica gel is immersed in water, at the ambient pressure water molecules enter the nanopores spontaneously. As the nanoporous space is filled, further increase in pressure would lead to only a negligible increase in water molecule density in the nanopores, since water is quite incompressible. Thus, no infiltration could be measured. As the silica is surface treated, chlorotrimethylsilane molecules diffuse into the nanopores and form a hydrophobic layer of $\mathrm{OSi}\left(\mathrm{CH}_{3}\right)_{3}$ groups through reaction with $\mathrm{OH}^{-}$groups. ${ }^{7}$ As the treatment time increases, more and more silane groups attach to nanopore wall and the surface coverage, $c$, keeps increasing. Eventually, nanopore surface transfers from wettable to non-wettable. Under this condition, water molecules cannot enter the nanopores spontaneously. A pressure must be applied so that the capillary effect can be overcome. In the current study, the infiltration pressure, $p_{\text {in }}$, is defined as the pressure at the onset of infiltration, which is dominated by the behaviors of water molecules in the largest nanopores. As $p_{\text {in }}$ is reached, water molecules are compressed into the nanoporous space, resulting in the formation of infiltration plateau in sorption isotherm curve. Because of the pore size distribution, the infiltration plateau is of a finite slope. When most of the nanopores are filled, the system compressibility is lost and the sorption isotherm curve becomes stiff. Upon unloading, owing to the difficulties in gas-phase nucleation and growth, ${ }^{8}$ the defiltration is much less pronounced compared with the infiltration. That is, the confined liquid tends to remain in the nanoporous silica gel at reduced pressure, even though it is nominally energetically unfavorable. Consequently, the excess solid-liquid interfacial energy cannot be released, which is the mechanism of energy absorption. Figure 1 shows clearly that as the treatment time, $t_{\mathrm{T}}$, is longer than $1 \mathrm{~h}$, the pore surface becomes hydrophobic. As $t_{\mathrm{T}}$ increases to $48 \mathrm{~h}, p_{\text {in }}$ rises to about $8 \mathrm{MPa}$. Further increase in $t_{\mathrm{T}}$ would not cause pronounced variation in $p_{\text {in }}$, indicating that the surface coverage has been saturated.

The infiltration volume, which can be taken as the width of the infiltration plateau, is about $0.83 \mathrm{~cm}^{3} / \mathrm{g}$, smaller than the gas-absorption measurement result, probably because of the reduction in accessible nanopore volume caused by infiltration of silane chains as well as the inaccessibility of smallest nanopores. It decreases slightly as the treatment time increases, which is in agreement with the increase in silane group density. However, the variation in infiltration volume is only secondary compared with the treatment time effect on the infiltration pressure. Thus, the change in energy absorption efficiency, which can be measured by the area enclosed by the loadingunloading loop, is nearly proportional to $p_{\text {in }}$.

Note that the $p_{\mathrm{in}}-t_{\mathrm{T}}$ relationship is highly nonlinear. As the critical treatment time is reached, initially the infiltration pressure increases rapidly with a small increment of $t_{\mathrm{T}}$. The $t_{\mathrm{T}}$ sensitivity of infiltration pressure decreases as $t_{\mathrm{T}}$ rises. When $t_{\mathrm{T}}>48 \mathrm{~h}$, the surface group density is saturated and further increase in $t_{\mathrm{T}}$ does not cause detectable changes in $p_{\text {in }}$. The nonlinearity of $p_{\mathrm{in}}-t_{\mathrm{T}}$ relationship should be attributed to that, as the surface coverage is relatively low, the silane groups attached on the nanopore surface are far and few between, and, therefore, they are relatively independent of each other. As the silane group density becomes increaseingly high, it is more and more difficult for chlorotrimethylsilane molecules to access untreated silica surface, and thus the increase in surface group density becomes slower.

After the first loading, since most of the porous space is occupied, at the second loading the infiltration volume is much smaller. From Figure 2, it can be seen that the sorption isotherm curves at the second loading are almost identical for all the samples, regardless of the large variation in surface treatment time. The limited infiltration occurs at relatively low pressure, nearly the same as the defiltration section of the sorption isotherm curve at the first loading. That is, while in most of the porous space the defiltration is difficult, there exists a reversible part of confined liquid, likely in the smallest accessible nanopores since the defiltration driven force is inversely proportional to the pore size. Since the specific energy involved in this process is much smaller than that in the first loading cycle, it does not have pronounced influence on the energy absorption performance. Moreover, since even when the silica gel is surface treated for $48 \mathrm{~h}$, the defiltration is still difficult, the uniformity of surface groups should not be a vital factor governing the defiltration behaviors.

In summary, through an experimental study, it was validated that the infiltration pressure of a nanoporous silica gel can be controlled in a broad range by adjusting the surface treatment time. As the treatment time is relatively short, the sensitivity of infiltration pressure is quite high; as the treatment time is relatively long, the sensitivity decreases and eventually the system behavior saturates. The energy involved at the second loading is negligible.

This work was supported by The Army Research Office under Grant No. W911NF-05-1-0288.

\section{References}

1 N. T. Nguyen, S. T. Wereley, Fundamentals and Applications of Microfluidics, Artech House Publ., 2006.

2 J. C. M. Li, J. Alloys Compd. 2000, 310, 24.

3 a) A. Han, Y. Qiao, J. Am. Chem. Soc. 2006, 128, 10348. b) X. Chen, F. B. Surani, X. Kong, V. K. Punyamurtula, Y. Qiao, Appl. Phys. Lett. 2006, 89, 241918. c) F. B. Surani, A. Han, Y. Qiao, Appl. Phys. Lett. 2006, 89, 093108.

4 E. Chaloner, Br. Med. J. 2005, 331, 119.

5 X. Kong, F. B. Surani, Y. Qiao, J. Mater. Res. 2005, 20, 1042

6 X. Kong, Y. Qiao, Appl. Phys. Lett. 2005, 86, 151919.

7 M. H. Lim, A. Stein, Chem. Mater. 1999, 11, 3285.

8 Y. Qiao, G. Cao, X. Chen, J. Am. Chem. Soc. 2007, 129, 2355. 